\title{
Identifying Map-Reading, Map-Using, and Map-Generating tasks in a crowdsourcing context
}

\author{
Claudia Robbi Sluter ${ }^{\mathrm{a}}$ *, João Vitor Meza Bravo ${ }^{\mathrm{b}}$ \\ ${ }^{a}$ Federal University of Rio Grande do Sul, Department of Geodesy, robbi.sluter@ufrgs.br \\ ${ }^{b}$ Federal University of Uberlândia, Institute of Geography, jvmbravo@gmail.com \\ * Corresponding author
}

Keywords: Map-reading, Map-using, Crowdsourcing mapping

\begin{abstract}
:
The literature has long addressed the map-reading tasks under a map-use context (Olson, 1976; Board, 1978; Van Elzakker, 2004). These researchers have used verbs for describing map-use actions, such as "search, locate, delimit, and identify." In the 1990s, GIS applications became popular, generating differences in map use research. Accordingly, Nyerges (1991) proposed considering the differences in map presentation in map use research under a computer-based perspective. Later, van Elzakker (2004) defined geographic tasks individuals usually perform while doing map-reading within a computer-based geographical exploration context. He defined geographic tasks similar to the map-reading tasks presented by Board (1978) around three decades earlier. However, a significant difference between these two approaches is the technological contexts they developed their research. While Board (1978) discussed map-reading tasks under a more traditional paper-based map use context, van Elzakker (2004) contributed to a computer-based map use context. We understand that the map-reading process under a crowdsourcing environment may also be different from a computer-based map.
\end{abstract}

Although many users do not have any cartographic training, they produce geoinformation in crowdsourcing platforms. As a result, users are sharing, generating, trusting, and commenting on geoinformation. Furthermore, individuals collaborating with VGI platforms have several kinds of motivation. Those different motivations lead to a different set of tasks they accomplish within these platforms. However, the main difference between the traditional map use and the crowdsourcing map use context is the possibility of individuals without education in cartography generate geoinformation.

Therefore, this paper addresses the question: What map-reading tasks do people perform on a crowdsourcing platform while using and generating geoinformation? Our research hypothesis is: If the crowdsourcing platform is used as a static reference map, there are no changes in map-reading tasks such as those associated with the traditional map use approach. However, if considered the technological developments, there are new map-reading tasks associated with (1) the technology itself and (2) the crowdsourcing map use context. Then, we considered that the map-reading tasks proposed by Board (1978) might be different due to the technological context we live in today.

We carried out two experiments. We accomplished the first experiment in a semi-controlled assisted environment (illumination, noises, equipment, contact with other people). The second experiment was online. We used the OpenStreetMap (OSM) as the crowdsourcing platform for operating the tasks in both experiments. For both experiments, the tasks are to create and disseminate geoinformation such as those we do on reference maps.

This paper describes the first experiment, which had eight (8) participants. The subjects were students from a Brazilian university enrolled with activities from two fields of knowledge: four (4) participants with education in Cartography and GIS and four (4) with education in Bioprocess and Biotechnology. The individuals frequently used maps for various purposes (navigation, exploration, and self-positioning) through smartphones, computers, and paper maps. Five (5) participants have already produced maps, at least once. We defined the experts' group by combining education in Cartography and GIS, expertise in producing maps, and research developed on Cartography and GIS. All participants were declared Portuguese native speakers or had Portuguese language proficiency certification. The test was designed and applied for Portuguese speakers because it is essential to avoid any semantic misunderstanding.

At the beginning of the test, we presented a scenario embedded in the crowdsourcing map context. The scenario consisted of a general situation of creating geoinformation about a place with where they are familiar. We invited the subjects to generate geoinformation within the OSM to present to some friends where they would give a party. We have read the following text to present the scenario: You will give a party to some friends. While inviting these friends, you have to present how to get to your chosen party venue. It would be best if you consider they have never been there before. We then asked the participants to answer what kind of product they usually use to solve this problem.

The result showed that the participants preferred to use maps when handling a situation proposed in the scenario. Few individuals have chosen to indicate the address by naming the street and describing where the party would occur. Most 
of them prefer to use some technological device for disseminating the party address. Even those who preferred to send the party address using texts commented that this action would occur through mobile devices, such as WhatsApp or Facebook messenger. Those participants whose preference was to use maps demonstrated the same inclination for disseminating the geoinformation using technological devices and the internet. Later, we asked the participants to write down a list that may represent the sequence of actions they should take to generate the map, and we reminded them to consider generating paper maps. This task required more attention than the first question of the test, and the individuals mentioned they were concerned about figuring out how to make a map. As a result, the participants have produced 29 distinct categories of actions to generate a paper map (Table 1).

For the third question, we have presented to the participants the OSM. Then, we asked them to list the sequence of actions they considered to generate the map by imagining using the OSM. Once again, they were not allowed to handle any device, and they should accomplish this task just by imagining the process. Along with some structural components, such as locating someplace, the participants have provided a detailed process slightly different from the previous task. The individuals produced 29 categories of actions, as we can see in Table 1. However, some of them are different from the first list. To highlight what is different, we have indicated New in the third column title. Finally, the individuals described the map production process after handling the OSM. We organized the results of the third task sequence in the fourth and fifth columns of the table. Besides giving us a mean for validating the results we achieved in the second task of the experiment, the individuals cited even more new map use tasks listed in the fifth column in Table 1.

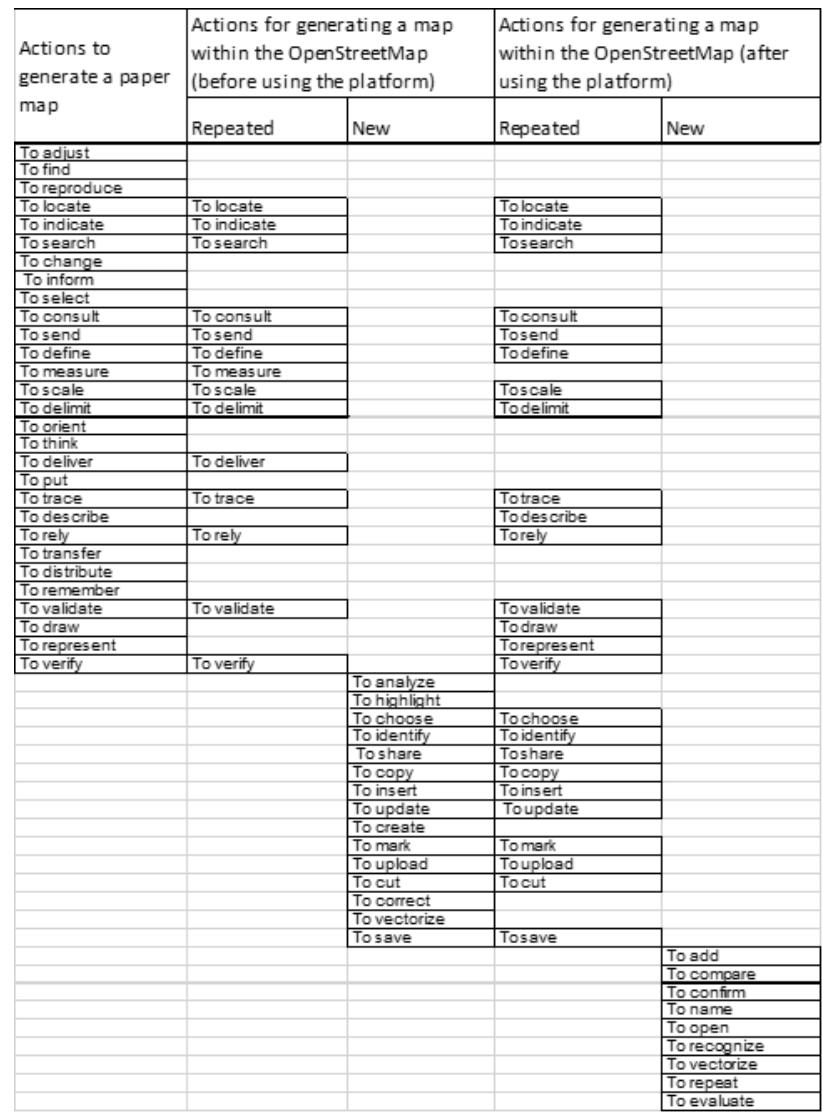

Table 1 - Map-reading, Map-using, and Map-generating tasks described by the individuals

We have found that technological developments have triggered such changes in map use during creating geoinformation. Based on the tests' results, we analyzed the role of the technology on the changes in map use promoted by the increasing use of geoinformation crowdsourcing platforms for disseminating geographic content. In conclusion, we confirmed our hypothesis that individuals perform new map-reading tasks under a crowdsourcing map use context.
BOARD, C. Map reading tasks appropriate in experimental studies in cartographic communication. Cartographica, vol. 15, 1978.

NYERGES, T. L. Analytical Map Use. Cartography and Geographic Information Systems, vol. 18, n. 1, 1991. pp. 11-22

OLSON, J. M. A Coordinate Approach to Map Communication Improvement. The American Cartographer, vol. 3, n. 2, 1976. pp. 151-160

VAN ELZAKKER, C. P. J. M. The Use of Maps in the Exploration of Geographic Data. Netherlands Geographical Studies 326, ITC, Utrecht/Enschede. 2004. 